\title{
Hamilton Cycles in Intersection Graphs of Bases of Matroids
}

\author{
Yinghao Zhang and Hongmei $\mathrm{Chi}^{*}$ \\ College of Science, Huazhong Agriculture University, Wuhan, China \\ ${ }^{*}$ Corresponding author
}

\begin{abstract}
The intersection graph of bases of a matroid $M=(E$, $B$ ) is a graph $G$ with vertex set $V(G)$ and edge set $E(G)$ such that $V(G)=B$ and $E(G)=B B^{\prime}$, where the same notation is used for the vertices of $G$ and the bases of $M, B$ and $B^{\prime}$ has no intersection. In this paper, we prove that for any given edge $e$ of $G$, the intersection graph $G$ of bases of a matroid $M$ with rank at least 2 has a Hamilton cycle containing edge $e$ and another Hamilton cycle avoiding edge $e$.
\end{abstract}

Keywords—matroid; intersection graph; Hamilton cycle

\section{INTRODUCTION}

Let $G$ be a graph with vertex set $V(G)$ and edge set $E(G)$. A matroid $M=(E, B)$ is a finite set $E$ together with a nonempty collection $B(M)$ of subsets of $E$ that satisfies the following condition: for any $B, B^{\prime} \mathrm{I} B(M)$ with $|B|=\left|B^{\prime}\right|$ and for any $e \mathrm{I} B \mid B^{\prime}$, there exists $e^{\prime} \mathrm{I} B \backslash B$ such that $(\{B \backslash e\}) \mathrm{E}\left\{e^{\prime}\right\} \mathrm{I} B(M)$. Each member of $B(M)$ is called a base of $M$. An element contained in every base is called a coloop, and an element contained in no base is called a loop. A matroid without loops and 2-circuits is called a simple matroid. The rank $r$ of a matroid is the number of elements in a base. We denote the uniform matroid of rank $m$ on an $n$-element set by $U_{m, n}$.

The base graph of a matroid $M=(E, B)$ is the graph $G^{\prime}=G^{\prime}(M)$ with vertex set $V\left(G^{\prime}(M)\right)=B(M)$ and edge set $E\left(G^{\prime}(M)\right)=\left\{B B^{\prime}: B\right.$, $B^{\prime} \mathrm{I} B(M)$ and $\left.\left|B \backslash B^{\prime}\right|=1\right\}$, where the same notation is used for the vertices of $G^{\prime}(M)$ and the bases of $M$. The basic properties and characterizations of base graphs of matroids can be found in [1] and [2].

Liu and Li [3-7] studied the properties of circuit graphs of matroids. The circuit graph of a matroid $M$ is a graph $G=G^{\prime \prime}(M)$ with vertex set $V=\left(G^{\prime \prime}(M)\right)=C(M)$ and edge set $E\left(G^{\prime \prime}\right)=\left\{C C^{\prime}\right.$ : $\mid C$ $\left.C C^{\prime}\right|^{1} 0, C, C^{\prime} \mathrm{I} C(M)$, where the same notation is used for the vertices of $G^{\prime \prime}(M)$ and the circuits of $M$. Next, we extend base graphs into a family of denser graphs by relaxing the requirement of vertices adjacency as follows: the intersection graph for bases of a matroid $M=(E, B)$ is the graph, denoted by $G^{I}(M)$ with vertex set $V\left(G^{I}(M)\right)=B(M)$ and edge set $E\left(G^{I}(M)\right)=\left\{B B^{\prime}:\left|B C \zeta B^{\prime}\right|^{1} \quad 0, B, B^{\prime} \mathrm{I} B(M)\right\}$.

For $r(M)=1$, the intersection of any two bases of $M$ is empty and thus the intersection graph $G^{I}(M)$ with rank $r(M)=1$ is a collection of $|B|$ isolated vertices. Clearly, for $r(M) \geqslant 2$, the intersection graph $G^{I}(M)$ contains the base graph $G^{\prime}(M)$ as a connected spanning subgraph. In particular, for $r(M)=2$, the intersection graph $G^{I}(M)$ is exactly the base graph $G^{\prime}$ of matroid $M$. The intersection graph $G^{I}(M)$ for bases of matroid $U_{2,4}$ is shown in Fig.1.

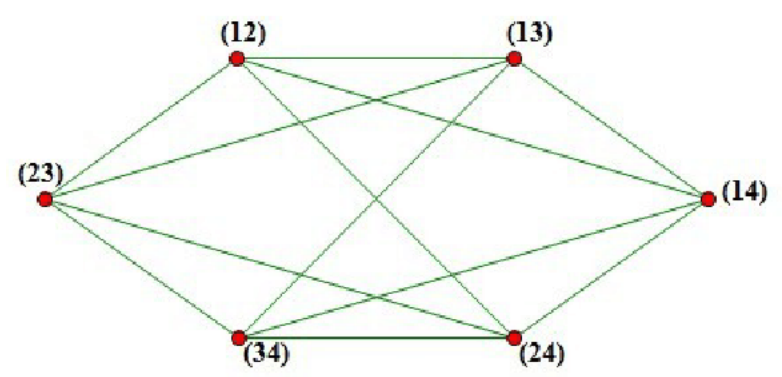

FIGURE I. THE INTERSECTION GRAPH OF MATROID $U_{2,4}$

The problem of Hamilton cycles in base graphs of matroids have been investigated by many researchers. Cummins [8] showed that the base graph of a matroid with at least three vertices has a Hamilton cycle. Bondy [9] showed not only that every base graph is Hamiltonian, but also that most are pancyclic. Holzmann and Harary [10] showed that for every edge in the base graph of a matroid there is a Hamilton cycle containing it and another Hamilton cycle avoiding it. In [11], we give the definition of intersection graph for bases of a matroid $M=(E, B)$ and showed that the intersection graph $G^{I}(M)$ for bases of a simple matroid $M$ with $\operatorname{rank} r(M) \geqslant 2$ has at least two edgedisjoint Hamilton cycles whenever $\left|V\left(G^{I}(M)\right)\right| \geqslant 5$.

Terminology and notations not defined here can be found in [12-13].

\section{The MAIN REsults}

In this paper, we prove that for any given $e \in E\left(G^{I}(M)\right)$, the intersection graph $G^{I}(M)$ of bases of a matroid $M$ with rank $r(M) \geqslant 2$ has a Hamilton cycle containing $e$ and another Hamilton cycle avoiding $e$ whenever $\left|V\left(G^{I}(M)\right)\right| \geqslant 4$. To prove the main theorems, we need the following lemmas. 
Lemma 1 [13] If $B_{1}$ and $B_{2}$ are bases of a matroid $M$ and $e \in B_{1} \backslash B_{2}$, then there exists an element $f \in B_{2} \backslash B_{1}$ such that both $\left(B_{1} \backslash\{e\}\right) \cup\{f\}$ and $\left(B_{2} \backslash\{f\}\right) \cup\{e\}$ are bases of M.

A graph $G$ is positively Hamiltonian, written $G \in H^{+}$, if for every edge of $\mathrm{G}$, there is a Hamilton circuit containing it. $\mathrm{G}$ is negatively Hamiltonian, written $G \in H^{-}$, if for every edge of G, there is a Hamilton circuit avoiding it. When $G \in H^{+}$and $G \in H^{-}$, we say that $\mathrm{G}$ is uniformly Hamiltonian.

Lemma 2 [10] Let $M=(E, B)$ be a matroid on $E$ and $G^{\prime}=G^{\prime}(M)$ be the matroid base graph of $M$. If $\left|V\left(G^{\prime}\right)\right| \geq 3$, then $G^{\prime}$ is positively Hamiltonian.

Lemma $3[10]$ Let $M=(E, B)$ be a matroid on $E$ and $G^{\prime}=G^{\prime}(M)$ be the matroid base graph of $M$. If $\left|V\left(G^{\prime}\right)\right| \geq 4$, then $G^{\prime}$ is negatively Hamiltonian.

In the following, we assume that matroid $M$ satisfies that $r(M)$ $\geqslant 2$ and $|B| \geq 3$. For any $e \mathrm{I} E \backslash B, B \mathrm{E}\{e\}$ contains a unique basic circuit, denoted by $C(e, B)$, and we use $B_{e}$ and $\bar{B}_{e}$ to denote the bases containing $e$ and avoiding $e$, respectively.

Lemma 4 Let $M=(E, B)$ be a matroid with $|E|=n$ and $r(M)=r$. If $M$ has no coloops, then $\left|\overline{B_{e}}\right| \geq n-r$ for any $e$ I $E$.

Proof Since $M$ does not have coloops, there exists a base $B$ of $M$ such that $e \ddot{\mathrm{I}} B$. For any element $f \hat{\mathrm{I}}(E \backslash B) \backslash\{e\}$, let $\mathrm{C}(f, B)$ I $B$ È $\{f\}$ be a basic circuit of $M$ with respect to the base $B$. Then exists an element $f^{\prime} \hat{\mathrm{I}} C(f, B) \backslash\{f\}$ such that $(B$ 任 $f\}) \backslash\left\{f^{\prime}\right\} \quad B(M)$ and $e \notin(B \cup\{f\}) \backslash\left\{f^{\prime}\right\}$. So there are at least $|(E \backslash B) \backslash\{e\}|+1=n-r$ bases of $M$ avoiding $e$.

Let $e \hat{I} E$ and let $G_{1}^{e}$ and $G_{2}^{e}$ be subgraphs of $G^{I}(M)$ induced by $\bar{B}_{e}$ and $B_{e}$, respectively. By the definition of intersection graphs, we have the following lemma immediately.

Lemma 5 Let $M=(E, B)$ be a matroid on $E$ and $G^{I}(M)$ be the intersection graph of bases of $M$. For $e$ I $E, G_{1}^{e}$ is the intersection graphs of bases of $M \backslash e$ and $G_{2}^{e}=G^{I}(M)$ $V\left(G_{1}^{e}\right)$.

It is easy to see that $G_{2}^{e}$ is a complete graph induced by the vertices containing $e$ and $\left|V\left(G_{2}^{e}\right)\right|=\left|B_{e}\right|$. The notations $G_{1}^{e}$ and $G_{2}^{e}$ keep the same meaning throughout the paper for a given $e$ I $E$.

Lemma 6 Let $M=(E, B)$ be a matroid on $E$ without coloops and with $\operatorname{rank} r(M) \geqslant 3$. Let $G^{I}(M)$ be the intersection graph of bases of $M$. For any edge $B_{1} B_{2} \in E\left(G^{I}(M)\right)$, there exists an

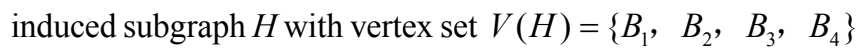

such that $B_{1} B_{2} \in E(H) \quad$ and $\quad\left\{B_{2}, B_{3}\right\} \subseteq V\left(G_{1}^{e}\right)$, $\left\{B_{1}, B_{4}\right\} \subseteq V\left(G_{2}^{e}\right)$ for some $e$ I $E$. Furthermore, $H$ is isomorphic to $K_{4}$.

Proof Note that $|E| \geq 4$ by the hypothesis. Obviously, if $|E|=n<2 r$, then $G^{I}(M) \cong K_{m}(m \geq 4)$ and thus the lemma holds. So we only need to consider the case that $|E|=n \geq 2 r \geq 6$. By the definition of intersection graph of bases of matroid and $B_{1} B_{2} \in E\left(G^{I}(M)\right)$, we have $1 \leq\left|B_{1} \cap B_{2}\right| \leq r-1$. Suppose that $\left|B_{1} \cap B_{2}\right|=r-1 \geq 2$. Then $\left|B_{1} \backslash B_{2}\right|=\left|B_{2} \backslash B_{1}\right|=1$. Set $B_{1}=A \mathrm{U}\{x\} \quad$ and $B_{2}=A \mathrm{U}\{y\} \quad$ (here $|A| \geq 2 \quad$ ). Since $r^{*}(M)=|E|-r(M) \geq 2 r-r \geq r$, there exists an element $b \in E \backslash\left(B_{1} \cup B_{2}\right)$ such that $C_{1}=C\left(b, B_{1}\right) \subseteq B_{1} \cup\{b\}$ and $C_{2}=C\left(b, B_{2}\right) \subseteq B_{2} \cup\{b\} \quad$. For every element $b \in E \backslash\left(B_{1} \cup B_{2}\right)$, if $\left(C_{1} \cup C_{2}\right) \cap A=\varnothing$, then either every element of $A$ is a coloop or $A=\varnothing$, which contradicts to the hypothesis of the lemma. So there exists an element $a \in A$ such that $a \in\left(C_{1} \cup C_{2}\right) \cap A$. We consider the following three cases.

Case $1 a \in\left(C_{1} \cap C_{2}\right)$.

It is obvious that $B_{4}=\left(B_{1} \cup\{b\}\right) \backslash\{a\}=(A \backslash\{a\}) \cup\{x, b\}$ and $B_{3}=\left(B_{2} \cup\{b\}\right) \backslash\{a\}=(A \backslash\{a\}) \cup\{y, b\}$ are bases of $M$. Thus there exists an element $e=x \in E$ such that $\left\{B_{2}, B_{3}\right\} \subseteq V\left(G_{1}^{e}\right),\left\{B_{1}, B_{4}\right\} \subseteq V\left(G_{2}^{e}\right)$.

Case $2 a \in C_{2} \backslash C_{1}$.

Clearly, $B_{4}=\left(B_{1} \cup\{b\}\right) \backslash\{a\}=(A \backslash\{a\}) \cup\{x, b\}$ is a base of $M$. Next we show that $y \notin C_{2}$ by contradiction. Suppose that $y \notin C_{2}$. Then $C_{2} \subseteq\left(B_{1} \cup B_{2}\right) \cup\{b\} \subseteq B_{1} \cup\{b\}$. But $C_{1} \subseteq B_{1} \cup\{b\}$ and there is only one circuit in $B_{1} \cup\{b\}$. So we have $C_{1}=C_{2}$, which is a contradiction. Set $B_{3}=\left(B_{2} \cup\{b\}\right) \backslash\{y\}=A \cup\{b\}$. Then there exists an element $e=x \in E$ such that $\left\{B_{2}, B_{3}\right\} \subseteq V\left(G_{1}^{e}\right),\left\{B_{1}, B_{4}\right\} \subseteq V\left(G_{2}^{e}\right)$.

Case $3 a \in C_{1} \backslash C_{2}$.

For this case, the arguments are similar to that of Case 2, and we can show that $x \in C_{1}$.

Thus obtain bases $B_{4}=\left(B_{1} \cup\{b\}\right) \backslash\{x\}=A \cup\{b\}$ and $B_{3}=\left(B_{2} \cup\{b\}\right) \backslash\{a\}=(A \backslash\{a\}) \cup\{x, b\}$. Then there exists an element $e=y \in E \quad$ such that $\left\{B_{2}, B_{3}\right\} \subseteq V\left(G_{1}^{e}\right)$, $\left\{B_{1}, B_{4}\right\} \subseteq V\left(G_{2}^{e}\right)$. Without loss of generality, we may assume $\left\{B_{2}, B_{3}\right\} \subseteq V\left(G_{1}^{e}\right),\left\{B_{1}, B_{4}\right\} \subseteq V\left(G_{2}^{e}\right)$ (if necessary, we can interchange $B_{1}$ and $B_{2}, B_{3}$ and $\left.B_{4}\right)$. 
Furthermore, it is easy to check that $\left(B_{1} \cap B_{2}\right) \backslash\{a\}=A \backslash\{a\} \subset B_{i}(i=\{1,2,3,4\})$, so the subgraph of $G^{I}(M)$ induced by $\left\{B_{1}, B_{2}, B_{3}, B_{4}\right\}$ is isomorphic to $K_{4}$ by the definition of intersection graph $G^{I}(M)$ of bases of matroid $M$.

Suppose that $1 \leq\left|B_{1} \cap B_{2}\right| \leq r-1$. Then there exists an element $e \in B_{1} \backslash B_{2}$. By Lemma 1 , there also exists an element $f \in B_{2} \backslash B_{1} \quad$ such that $B_{3}=\left(B_{1} \backslash\{e\}\right) \cup\{f\} \quad$ and $B_{4}=\left(B_{2} \backslash\{f\}\right) \cup\{e\}$ are bases of matroid $M$. Then $\left\{B_{2}, B_{3}\right\} \subseteq V\left(G_{1}^{e}\right),\left\{B_{1}, B_{4}\right\} \subseteq V\left(G_{2}^{e}\right)$.

It is not hard to see that the subgraph induced by $\left\{B_{1}, B_{2}, B_{3}, B_{4}\right\} \quad$ is also isomorphic to $K_{4}$ because $\left(B_{1} \cap B_{2}\right) \subset B_{i} \quad(i=\{1,2,3,4\})$. Hence, for any edge $B_{1} B_{2} \in E\left(G^{I}(M)\right)$, we can find an induced subgraph $H$ with vertex set $V(H)=\left\{B_{1}, B_{2}, B_{3}, B_{4}\right\}$ such that such that $B_{1} B_{2} \in E(H)$ and $\left\{B_{2}, B_{3}\right\} \subseteq V\left(G_{1}^{e}\right), \quad\left\{B_{1}, B_{4}\right\} \subseteq V\left(G_{2}^{e}\right)$ for some $e$ I $E$. Furthermore, $H$ is isomorphic to $K_{4}$.

Now we proceed to the main theorems.

Theorem 1 Let $M=(E, B)$ be a matroid without coloops on $E$ and $G=G^{I}(M)$ be the intersection graph of bases of $M$. If $\left|V\left(G^{I}(M)\right)\right| \geq 3$, then $G^{I}(M)$ is positively Hamiltonian.

Proof We prove the theorem by induction on $|E|=n$. Note that we have $|E| \geq 3$ and $r(M) \geqslant 2$. If $|E|=3$ and $r(M)=2$, then $M \cong U_{2,3}$ and it is easy to see that $G=G^{I}(M) \cong K_{3}$, so the theorem holds. Suppose that the result is holds for $|E| \leq n-1$. We prove that the result also holds for $|E|=n>3$. Obviously, if $|E|=n<2 r$, then $G=G^{I}(M) \cong K_{m}(m \geqslant 3)$ and thus the theorem holds.

In fact, if $r(M)=2$, then the intersection graph $G=G^{I}(M)$ is isomorphic to the matroid base graph of $M$. The theorem holds by Lemma 2 . So we only need to consider the case that $\mathrm{r}(\mathrm{M}) \geqslant 3$ and $|E|=n \geqslant 2 r \geqslant 6$. Let $B_{1} B_{2}$ be any edge of $G^{I}(M)$.

By Lemma 6 , for any edge $B_{1} B_{2} \in E\left(G^{I}(M)\right)$, we can find an induced subgraph $H$ with vertex set $V(H)=\left\{B_{1}, B_{2}, B_{3}, B_{4}\right\}$ such that such that $B_{1} B_{2} \in E(H)$ and $\left\{B_{2}, B_{3}\right\} \subseteq V\left(G_{1}^{e}\right),\left\{B_{1}, B_{4}\right\} \subseteq V\left(G_{2}^{e}\right)$ for some $e$ I $E$. Furthermore, $H$ is isomorphic to $K_{4}$. By Lemma 4, we have $\left|V\left(G_{1}^{e}\right)\right|=\left|\overline{B_{e}}\right| \geqslant \mathrm{n}-\mathrm{r} \geqslant \mathrm{r} \geqslant 3$. If $M \backslash e$ has coloops, then $G_{1}^{e}$ is a complete graph $K_{m}$ with order $\mathrm{m} \geqslant 3$. So $G_{1}^{e}$ has a Hamilton cycle $C_{1}$ containing $B_{2} B_{3}$. If $M \backslash e$ does not have coloops, then the induction hypothesis assures the existence of a Hamilton cycle $C_{1}$ of $G_{1}^{e}$ containing $B_{2} B_{3}$. It is easy to see that $G_{2}^{e}=$
$G^{I}(M)-V\left(G_{1}^{e}\right)$ is a complete graph and thus there is a Hamilton path $P$ in $G_{2}^{e}$ connecting $B_{1}$ and $B_{4}$. Thus $C=\left(C_{1}-B_{2} B_{3}\right) \cup B_{3} B_{4} \cup P \cup B_{1} B_{2}$ is a Hamilton cycle which containing $B_{1} B_{2} \in E\left(G^{I}(M)\right)$ (see Fig. 2). The proof is complete.

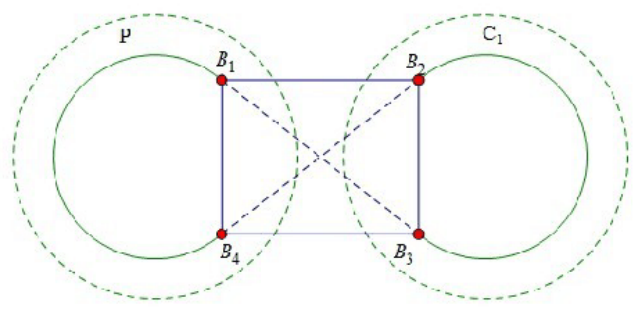

FIGURE II. HAMILTON CYCLE CONTAINING $B_{1} B_{2}$

Theorem 2 Let $M=(E, B)$ be a matroid without coloops on $E$ and $G=G^{I}(M)$ be the intersection graph of bases of $M$. If $\left|V\left(G^{I}(M)\right)\right| \geq 4$, then $G^{I}(M)$ is negatively Hamiltonian.

Proof If $r(M)=2$, then the intersection graph $G^{I}(M)$ of bases of $M$ is isomorphic to the base graph $G^{\prime}(M)$ of $M$ and thus the theorem holds by Lemma 3. Next we assume that $r(M) \geqslant 3$. Suppose that $|E|=4$ or $|E|=5$ and $r(M)=3$. Then the intersection graph $G^{I}(M)$ is a complete graph $K_{m}$ with order $m \geqslant 4$, the theorem clearly holds. Moreover, if $|E|=n<2 r$, then $G^{I}(M)$ $\cong K_{m} \quad(m \geqslant 3)$ and so the theorem also holds. Now we consider the remaining case that $r(M) \geqslant 3$ and $|E|=n \geqslant 2 r \geqslant 6$. Let $B_{1} B_{2}$ be any edge of $G^{I}(M)$. By Lemma 6, for any edge $B_{1} B_{2} \in E\left(G^{I}(M)\right)$, we can find an induced subgraph $H$ with

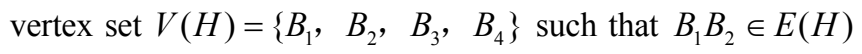
and $\left\{B_{2}, B_{3}\right\} \subseteq V\left(G_{1}^{e}\right),\left\{B_{1}, B_{4}\right\} \subseteq V\left(G_{2}^{e}\right)$ for some $e \dot{\mathrm{I}} E$. Furthermore, $H$ is isomorphic to $K_{4}$. In fact, the induced subgraph $H$ is isomorphic to $K_{4}$ implies that $B_{1} B_{3} \in E\left(G^{I}(M)\right)$ and $B_{2} B_{4} \in E\left(G^{I}(M)\right)$.

By Lemma 4, we have $\left|V\left(G_{1}^{e}\right)\right|=\left|\overline{B_{e}}\right| \geqslant \mathrm{n}-\mathrm{r} \geqslant \mathrm{r} \geqslant 3$. If $M \backslash e$ has coloops, then $G_{1}^{e}$ is a complete graph $K_{m}$ with order $\mathrm{m} \geqslant 3$. So $G_{1}^{e}$ has a Hamilton cycle $C_{1}$ containing $B_{2} B_{3}$. If $M \backslash e$ does not have coloops, then by Theorem 1, there exists a Hamilton cycle $C_{1}$ of $G_{1}^{e}$ containing $B_{2} B_{3}$.

Obviously, $G_{2}^{e}=G^{I}(M)-V\left(G_{1}^{e}\right)$ is a complete graph and there is a Hamilton path $P$ in $G_{2}^{e}$ connecting $B_{1}$ and $B_{4}$. Thus $C^{\prime}=\left(C_{1}-B_{2} B_{3}\right) \cup B_{1} B_{3} \cup P \cup B_{4} B_{2}$ is a Hamilton cycle avoiding $B_{1} B_{2} \in E\left(G^{I}(M)\right)$ (see Fig. 3). 


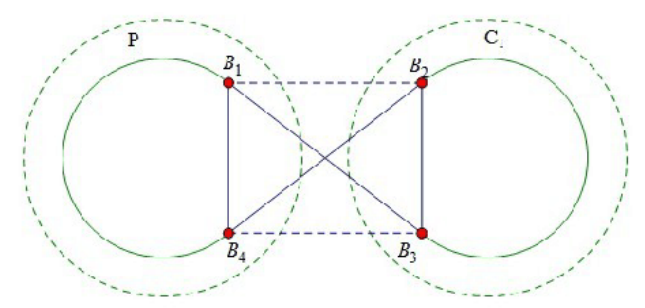

FIGURE III. HAMILTON CYCLE AVOIDING $B_{1} B_{2}$

Corollary 1 Let $M=(E, B)$ be a matroid on $E$ and $\mathrm{G}=G^{I}(M)$ be the intersection graph of bases of $M$. If $\left|V\left(G^{I}(M)\right)\right| \geq 4$, then $G^{I}(M)$ is uniformly Hamiltonian.

Remark Note that the results in this paper are best possible in the condition $\left|V\left(G^{I}(M)\right)\right| \geq 4$ in the theorem is necessary. In fact, if $\left|V\left(G^{I}(M)\right)\right|<3$, then $G^{I}(M)$ has no cycles; if $\left|V\left(G^{I}(M)\right)\right|=3$, then $G^{I}(M)$ is isomorphic to 3-cycle which is not negatively Hamiltonian.

\section{ACKNOWLEDGMENT}

This work was supported by grants from National Natural Science Foundation of China (No. 31601209), Natural Science Foundation of Hubei province (No. 2017CFB398).

\section{REFERENCES}

[1] S B Maurer, "Matroid basis graph I," J. of Combin. Theory Ser. B, vol.14, pp. 216-240, 1973.

[2] S B Maurer, "Matroid basis graph II," J. of Combin. Theory Ser. B, vol.15, pp. 121-145, 1973.

[3] P Li, G Liu, "The edge connectivity of circuit graphs of matroids," International Conference on Computational Science 2007, Part III, LNCS 4489. Berlin: Springer-Verlag, 2007, pp. 440-443.

[4] P Li, G Liu, "Cycles in matroid circuit graphs," Graphs and Combinatorics, vol. 23, pp. 425-431, 2007.

[5] G Liu, P Li, "Paths in circuit graphs of matroids," Theoretical Computer Science, vol. 23 pp. 425-431, 2007.

[6] P Li, G Liu, "Hamiltonian cycles in matroid circuit graphs," Computers and Mathematics with Applications, vol. 55, pp.654-6592008.

[7] P Li, G Liu, "The connectivity and minimum degree of circuit graphs of matroids," Acta Mathematica Sinica, English Series, vol. 26, pp. 353-360, 2010.

[8] R L Cummins, "Hamiltonian circuits in tree graphs," IEEE Trans. Circuits Syst, vol.13, pp. 82-90, 1966.

[9] J A Bondy, "Pancyclic graphs: II," Proceedings of the Second Louisiana Conference on Combinatorics, Graph Theory and Computing. Boton Rouge: AMS, pp.167-172, 1971.

[10] C A Holzmann, F Harary, “On the tree graph of a matroid," SIAM J Appl. Math., vol.22, pp.187-193, 1972.

[11] Y Zhang, Q Yu G Liu, "Edge disjoint Hamilton cycles in intersection graphs of bases of matroids," Utilitas. Math., vol. 90, pp.327-334, 2013.

[12] J G Oxley, Matroid Theroy. New York: Oxford University Press, 1992.

[13] J A Bondy, U S R Murty, Graph Theory with Applications, New York: Macmillan Press, 1976. 